\title{
Pinte o 7: Sistema Interativo para o ensino de progra- mação de computadores para o público infanto-juvenil
}

\author{
Márcio de Lima Passos¹, Angela Lima Peres², Mozart Melo ${ }^{3}$ \\ Centro de Estudos Superiores de Maceió - CESMAC \\ CEP: 57.051-160, Maceió-AL, Brasil \\ marciopassosbel@gmail.com, limaperes@gmail.com, mozartkmflgmail.com
}

\begin{abstract}
This paper is about an interactive system for teaching computer programming to kids and youngsters from the 8th grade up. Follow from this goal, the system aims to promote the learning of computer logic through an easy-to-use tool based on usability recommendations. Also, available similar tools are studied in the development process. To validate the solution an applied test session was thrown at middle school students in Maceió - AL. Therefore the system hopes to teach logic connections for students to apply in any other field of study. The tool is a free open web technologies based solution, released as free software under the MIT License.
\end{abstract}

Resumo. Apresenta-se o Pinte o 7, uma proposta de sistema interativo como ferramenta para o ensino de programação ao público infanto-juvenil. Buscase promover o aprendizado da lógica computacional por meio da facilitação da interatividade usuário-ferramenta com base em heurísticas de usabilidade. Verifica-se ferramentas existentes disponiveis na rede. Valida-se a ferramenta a partir de uma experimentação inicial com estudantes de uma escola de Ensino Fundamental e Médio na cidade de Maceió-AL. Espera-se que o ensino a partir da ferramenta, reflita conexões lógicas a serem aplicadas a quaisquer outras disciplinas. A ferramenta é solução nacional, de código aberto, e baseada em tecnologias livres.

\section{Cenário de Uso}

Um estudo feito pela Associação Brasileira de Empresas de Tecnologia da Informação e Comunicação (BRASSCOM, 2013) relata que, ainda em 2014, o Brasil terá uma demanda de 78 mil profissionais para trabalhar no setor de novas tecnologias; porém, a oferta será de apenas 33 mil recém-formados de áreas correlatas à computação.

Mark Surman, Diretor da Mozilla, afirma em entrevista para Romani (2013), que essa deficiência digital poderia ser suprida caso o ensino de programação fosse iniciado nas escolas, que ele denomina de 'Alfabetização Digital'; posto que, atualmente, o aprendizado de programação de computadores se faz tão importante quanto a escrita e a leitura fizeram a seu tempo.

Algumas soluções para a debilidade mencionada já vêm sendo propostas há alguns anos no intuito de dar suporte ao aprendizado de algoritmos e fundamentos de programação de computadores ao público jovem. E ainda, tal aprendizado se faz por 
ferramentas que encontram-se disponíveis na rede, como por exemplo o Khan Academy ${ }^{1}$, o Scratch $^{2}$, o Isla language ${ }^{3}$, e a linguagem LOGO ${ }^{4}$.

Dentre tais ferramentas citadas acima, distintas, e em suas especificidades, pode ressaltar-se suas formas de interação, colaboração, plataformas, linguagens, tecnologias, e versões. Ao embasar-se nas definições, funções, e características destas ferramentas, releva-se ser cada vez mais imprescindível, o uso correto de heurísticas de usabilidade, para propiciar um processo de ensino-aprendizagem satisfatório e eficaz, tanto para os pequenos atores, quanto para os educadores.

No Brasil também já existem pesquisas acerca do ensino-aprendizado de raciocínio computacional, como por exemplo, o projeto Scalable Game Design - Brasil (SGB-BR $)^{5}$, desenvolvido pelo Grupo de Pesquisa de Engenharia Semiótica da Pontifícia Universidade Católica do Rio de Janeiro, que busca desenvolver tecnologias nacionais que estimulem e apóiem o ensino-aprendizado de Computação no ensino fundamental e médio no país.

Apresenta-se uma proposta de sistema interativo designado ao ensino de algoritmos e fundamentos básicos de programação para jovens do ensino fundamental e médio, desenvolvido com base nas heurísticas de usabilidade de Jakob Nielsen (1993), e nas características da linguagem LOGO.

\section{Desenvolvimento}

O Pinte o 7, ou P7, roda no navegador do usuário e utiliza a linguagem de marcação HTML5, PHP (CodeIgniter) e JavaScript (jQuery/jQueryUI), juntamente com os recursos de apresentação das CSS3 (Cascading Style Sheets). A ferramenta segue os padrões de desenvolvimento do W3C, com código semântico e separação total das camadas de estrutura, conteúdo, apresentação, estilos e interatividade, utilizando JavaScript não-obstrutivo.

Para a escolha dos ícones foi usado o pacote WebHostingHub Glyphs, lançado pela WebHostingHub (http://www.webhostinghub.com) sob a licença Creative Commons 3. O P7 é baseado na linguagem LOGO, e utiliza o interpretador escrito em JavaScript - Papert, desenvolvido por Thomas Figg e publicado com código aberto no repositório colaborativo Google Code.

A ferramenta está em sua terceira versão. A primeira foi desenvolvida como um protótipo, contendo apenas algumas funcionalidades básicas de um sistema para ensino de programação. Na segunda, foi incorporado o interpretador LOGO e novo leiaute e

\footnotetext{
${ }^{1}$ Disponível em: http://www.khanacademy.org. Acesso em: 01/07/14.

${ }^{2}$ Disponível em: http://scratch.mit.edu/. Acesso em: 01/07/14.

${ }^{3}$ Disponível em: http://islalanguage.org/. Acesso em: 01/07/14.

${ }^{4}$ Disponível em: http://el.media.mit.edu/logo-foundation/index.html. Acesso em: 01/07/14.

${ }^{5}$ Disponível em: http://www.serg.inf.puc-rio.br/wiki/index.php/Página_principal. Acesso em: 01/07/14.
} 
funcionalidades. Na versão atual, foram revistas algumas características, performance e usabilidade do Pinte o 7.

O Pinte o 7 foi validado por um público de 20 estudantes infanto-juvenis, com média de idade de 15 anos, com baixo ou nenhum conhecimento de programação, em uma escola de ensino médio em Maceió, Alagoas. Tais dados foram obtidos mediante um questionário respondido pelos alunos também com seus dados pessoais e gostos relacionados às disciplinas escolares. As respostas estão disponíveis na rede e podem ser conferidas pelo apontador http://linkparaasrespostas.com.

A validação iniciou-se se com a apresentação de um vídeo acerca da importância do ensino de programação nos dias de hoje - "What most schools don't teach", veiculado pelo sítio code.org na rede. Seguiu-se com uma introdução sobre computador, linguagens de programação, algoritmos, a linguagem LOGO e alguns de seus comandos básicos, além do Pinte o 7, no qual o professor explicava os comandos e conceitos relacionados ao correto manuseio da ferramenta para execução dos exercícios: desenhar um quadrado usando comandos simples; desenhar um quadrado usando estruturas de repetição; desenhar um quadrado usando funções; e, desenhar uma estrela de tamanho variável passando pelo argumento de uma função. A cada execução, ao aluno clicar em Compartilhar e preencher uma caixa de texto com seu nome seguido pelo número do exercício resolvido, eram gravados arquivos com Seu nome, data e hora de criação do arquivo; e ao final, eram apresentados à solução proposta.

Fato importante para a usabilidade, destaca-se quando, antes mesmo das instruções do professor, alguns alunos por interesse próprio, já observaram e iniciaram sua interação; tal direcionamento da atenção primária do aluno, se fez por possibilitar a escolha e/ou troca do personagem de seu perfil, o que gerava automaticamente uma identificação do aluno com a ferramenta, ao eles identificarem-se com um personagem.

Outra situação observada, foi um aluno que não seguia as instruções do professor e os exercícios propostos, mas interagiu com a ferramenta por meio de experimentações livres, buscando conhecer no menu Ajuda novos jeitos para desenhar e criar estruturas, o que lhe permitiu criar maior intimidade com a ferramenta, a ponto de ajudar na dúvida de outros alunos. Motivo que o considerou o aluno que obteve maior desempenho da turma, apesar dos erros cometidos na programação dos exercícios propostos.

\section{Apresentação}

Na Figura 1 abaixo, apresenta-se a atual versão 3.0 do P7, na qual demonstra-se a tela principal do aplicativo, na qual destaca-se, na interface, a área livre central, que tem o objetivo de prender a atenção do aluno no mais importante: o aprendizado. Nesta área, é visualizado o resultado dos comandos acionados.

Ainda na Figura 1 mostrada a seguir, pode-se observar nas demais áreas identificadas por numerais: 


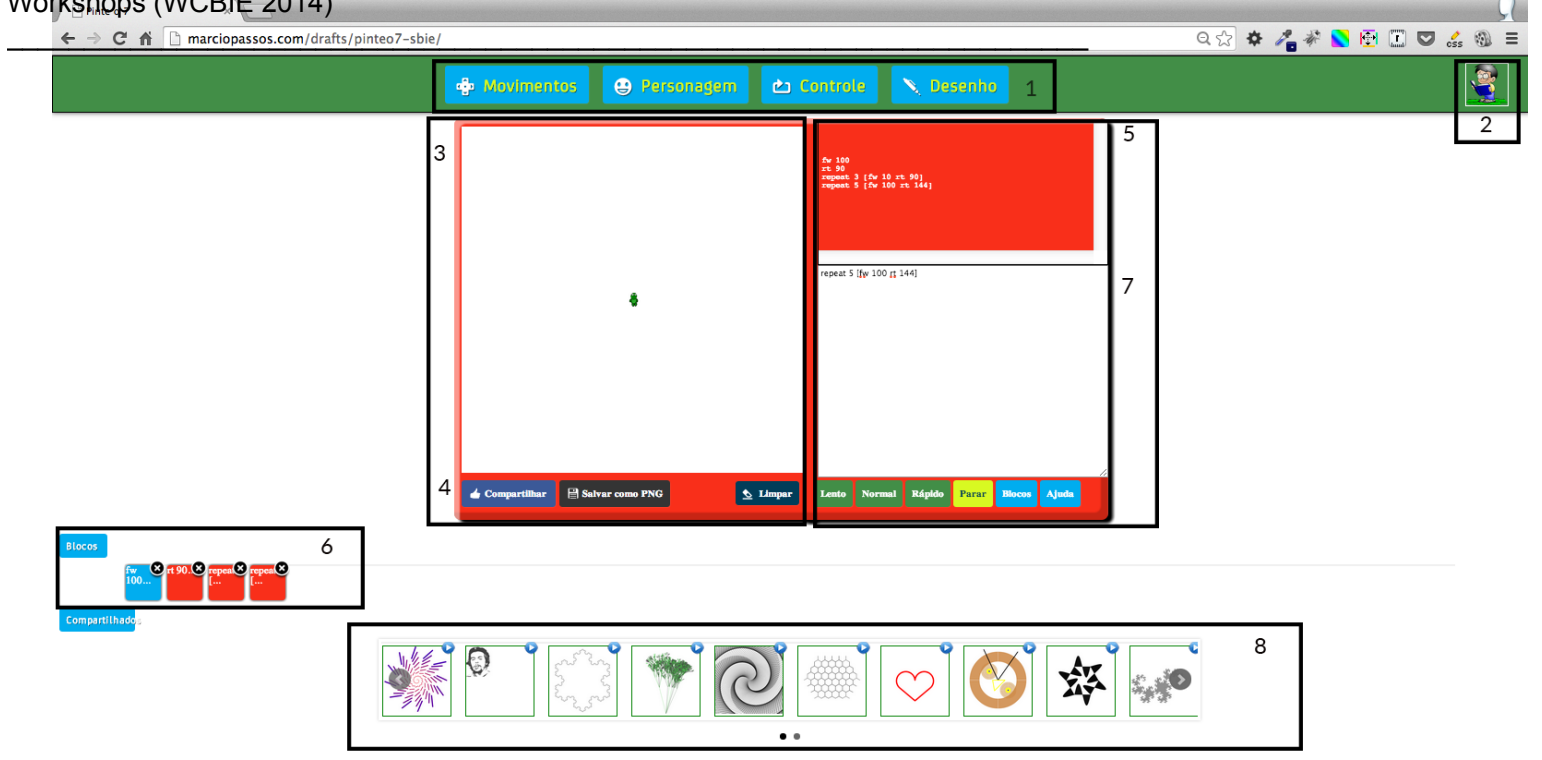

Figura 1. Tela Principal da Ferramenta

1. Menu principal: facilita a interação com o público mais jovem, e procura obedecer as heurísticas de design minimalista, falar a linguagem do usuário, e minimizar a carga de memória. As funcionalidades básicas são agrupadas nas opções principais para este público, que consistem na escolha do personagem, no desenho de figuras, e no movimento. Após o ensino dos comandos e exercícios básicos, o acionamento de estruturas de controle permitirão desenvolver exercícios mais avançados.

2. Perfil: a figura do aluno está relacionada ao perfil escolhido para a utilização do aplicativo. O perfil de professor provê outras possibilidades de interação, onde a ferramenta deve oferecer uma base de exercícios, com conceitos associados e possibilidades pedagógicas de cada um destes exercícios.

3. Canvas: área principal do aplicativo, onde o personagem executa os comandos do usuário.

4. Opções do Canvas: proporciona o compartilhamento da imagem gerada pelo aluno, salvar a imagem gerada no computador do usuário, e limpar (renovar) a imagem do canvas.

5. Memória do programa atual: grava todos os comandos digitados pelo usuário na interação atual, ficando disponíveis para consulta; o que permite, por exemplo, revisar os conceitos apresentados nos exercícios anteriores, repetir a execução, e relacionar com os novos aprendizados.

6. Blocos-Memória: toda vez que um código é executado, se este código é diferente de todos os outros já executados por este usuário, então é gerado um bloco; este bloco (conjunto de instruções) pode ser recarregado na linha de comando, reposicionado na interface de blocos, ou excluído do blocos-memória.

7. Linha de Comando: permite interação de forma direta usuário-aplicativo, ao digitar o código do seu programa e escolher um modo de execução; por exemplo: lento - executa o desenho em velocidade baixa, mostrando o personagem; normal - executa o código em velocidade moderada e não mostra o persona- 
gem durante o desenho; rápido - executa o programa, gera a imagem rapidamente e não mostra o personagem; parar - termina a execução atual; blocos troca a interface de linha de comando pela interface de blocos; ajuda - mostra comandos LOGO suportados pelo P7 com exemplos. Independente da escolha, é garantido que o aluno execute o programa e aguarde até o final de seu processamento; assim, a ferramenta assegura que se visualize os resultados do código executado antes de reiniciar um outro procedimento; o que proporciona um aprendizado mais efetivo.

8. Programas Compartilhados: os códigos compartilhados são armazenados no servidor e exibidos, junto com as imagens geradas, a todos os usuários

Respeitando as heurísticas propostas por Nielsen (1993), destaca-se a presença constante da ajuda ao usuário (legendas ${ }^{6}$ e documentação) a um clique. Na ferramenta também propõe-se a interação por blocos, com a qual o aluno pratica os conceitos básicos de funções e modularização, ao criar códigos de maneira personalizada e poder arrastá-los e organizá-los como desejar. A tela de blocos é acionada através do clique no botão "Blocos".

Apesar de no estágio atual de desenvolvimento, a ferramenta possibilite criar programas simples de forma facilitada por meio dos diversos tipos de interação oferecidos, a interface de programação visual, apresentada na Figura 2 a seguir, demonstra a necessidade de outros tipos de controles para que esta venha a ser realmente eficiente ao usuário.

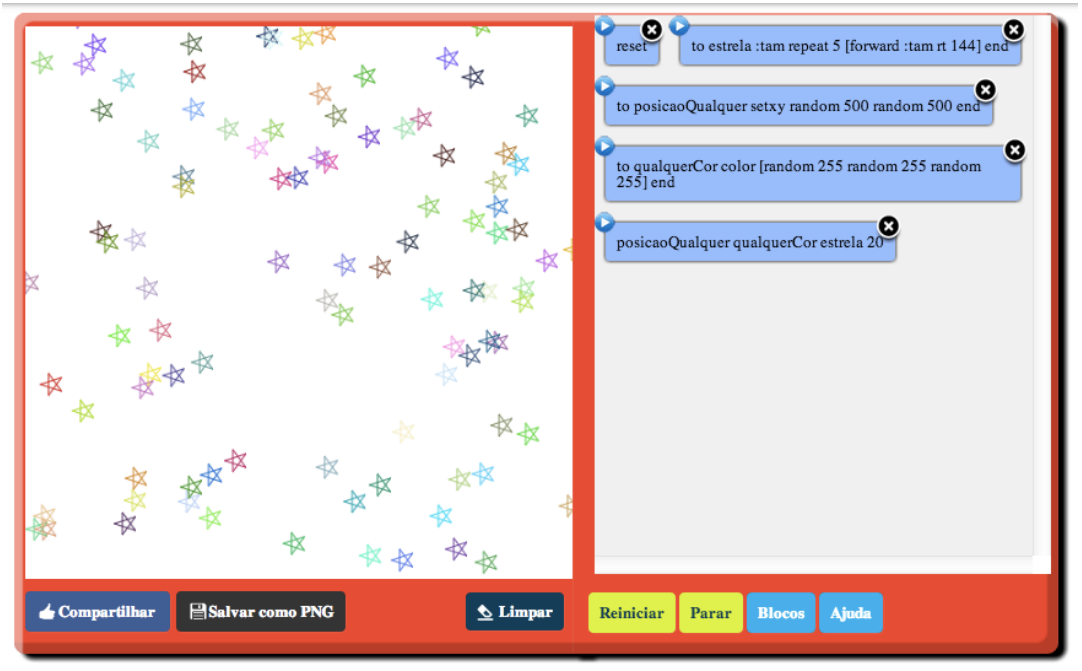

Figura 2. Modo Blocos

Por ser uma ferramenta com um interpretador LOGO, a Interatividade dá ao programador resposta imediata às suas instruções, como na Figura 3 a seguir, onde mostra-se a resposta ao usuário no envio correto do comando "FORWARD 100": o personagem move 100 passos à frente.

\footnotetext{
${ }^{6}$ Disponível em: http://www.techterms.com/definition/tooltip. Acesso em: 01/07/14.
} 


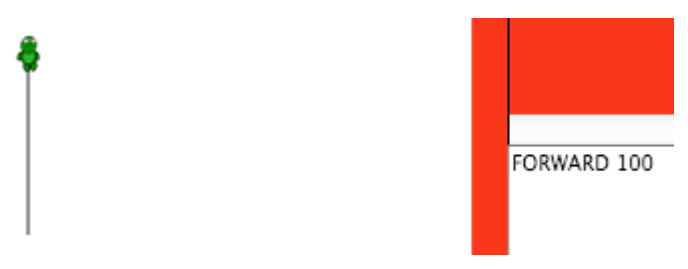

Figura 3. Resposta ao comando do usuário no P7

Tal interatividade auxilia na procura por erros de código (debug) e consequentemente, no processo de aprendizagem. Como exemplifica-se na Figura 4 a seguir, com respostas da ferramenta ao usuário, no caso de um problema de digitação do comando "FOWAD", que não é um comando LOGO primitivo original ou procedimento que o usuário definiu; ou no caso do problema ser um comando incompleto "FORWARD", onde o interpretador reconhece a palavra, porém para executa-lá precisa de um complemento ao comando. Ressalta-se a homogeneidade das mensagens de erro exibidas na tela, característica fundamental à usabilidade.

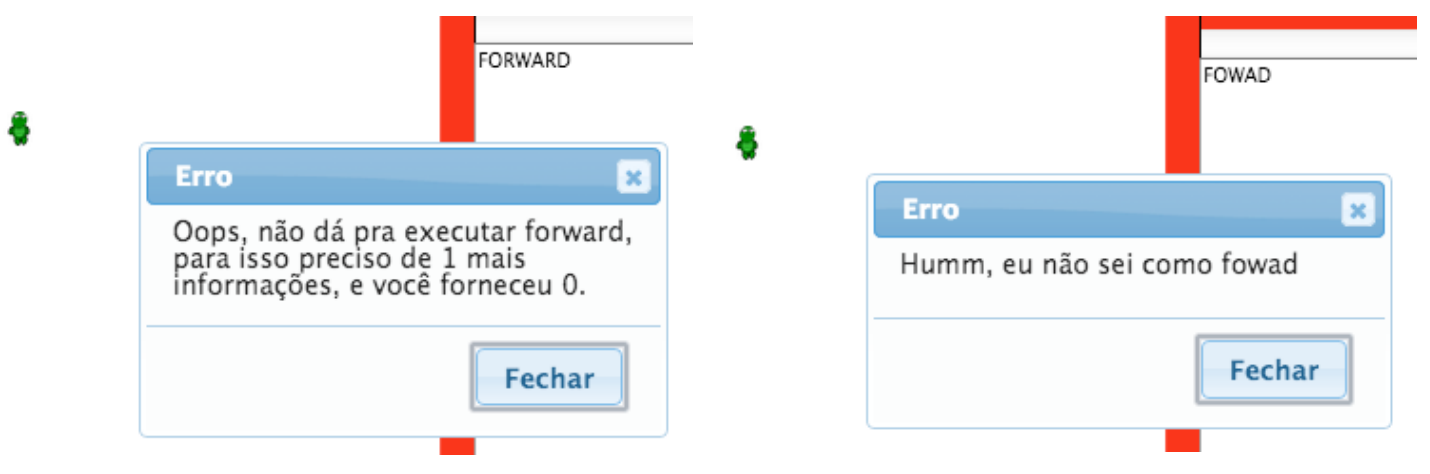

Figura 4. Interatividade nas respostas a comandos do usuário no P7

No que diz respeito à Modularidade ou Extensibilidade, programas em LOGO são geralmente uma coleção de regras ou procedimentos escritos na área de linha de comando da ferramenta ou através do menu principal. Estes procedimentos, regras ou módulos de regras, e funções, são escritos utilizando a palavra reservada 'to' seguida pelo nome do procedimento e seus argumentos, e as linhas de código que definem a função. A palavra reservada 'end' é usada para sinalizar o final de um procedimento.

No programa a seguir, começamos definindo um procedimento para desenhar um quadrado. Em seguida, o usamos como sub-procedimento em outro procedimento. Adicionamos mais um para levar o personagem a uma posição aleatória no canvas (500x500 pixels) e outro para mudar a cor do traço deixado pelo personagem. E então, analogamente poderíamos construir algo maior. Demostramos tal programa na Figura 5 
abaixo, que mostra o resultado do código fonte executado na ferramenta Pinte 7 .

to quadrado

repeat 4 [forward 30 right 90 ]

end

to flor

repeat 36 [right 10 quadrado]

end

to posicaoQualquer

setxy random 500 random 500

end

to corQualquer

color [random 255 random 255 random 255]

end

to jardim

repeat 50 [corQualquer posicaoQualquer flor] end

jardim

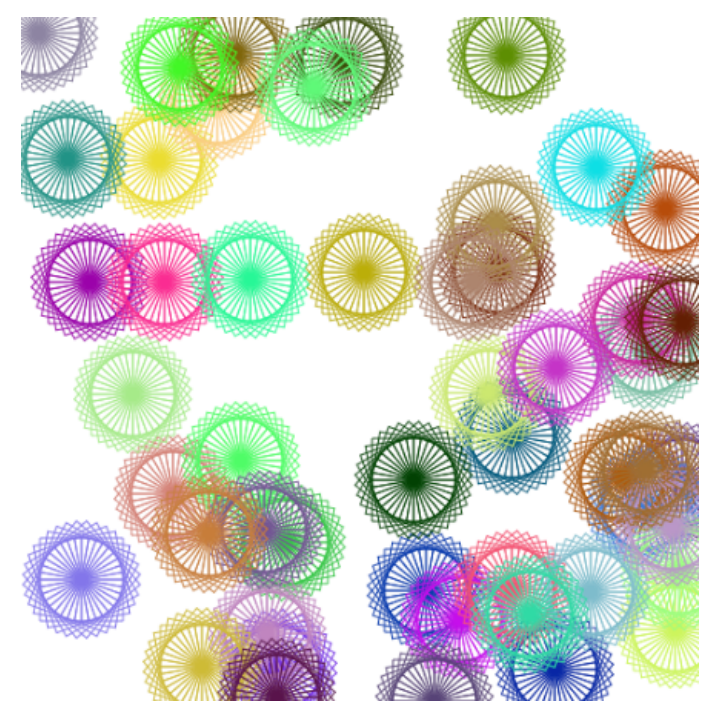

Figura 5. Detalhes de código fonte LOGO e imagem gerada no P7

Assim que um procedimento é definido, passa a ser tratado como uma palavra nova; a programação é feita de modo a adicionar palavras ao vocabulário do interpretador, ensinando ao LOGO palavras novas, formadas por palavras que ele já conhece, em um processo semelhante ao de aprendizagem da fala.

Em termos de Flexbilidade, a linguagem trabalha com palavras e listas. Uma palavra em LOGO é uma cadeia de caracteres. Uma lista é uma coleção ordenada de palavras e/ou listas. Números são palavras, e são especiais pois, pode-se utilizá-los em operações matemáticas. Neste sentido, a linguagem LOGO auxilia o usuário, como demonstra-se no exemplo a seguir:

print $13+1$

saída: 14

E pode-se ainda, criar procedimentos ou funções recursivas para cálculo de fatorial, como a apresentada abaixo:

to fatorial :numero

if : numero $=1$ [output 1]

output :numero * fatorial :numero - 1

end

print fatorial 3

saída: 6

As tecnologias utilizadas são livres, o código fonte do aplicativo encontra-se compartilhado no Github, repositório para colaboração na rede, onde todo o processo de desenvolvimento é documentado através de tickets e wikis, e pode ser acessado no apontador: www.github.com/mlpassos/pinteo7. A licen- 
ça é a padrão do repositório, do Instituto de Tecnologia de Massachusetts - MIT para software livre. Dessa forma, o sistema é mantido pelos desenvolvedores e tem sua construção documentada por eles e por qualquer usuário que deseje relatar algum problema.

\section{Considerações Finais}

Com o resultado da validação e os dados de acertos - respectivamente $96 \%$, $89 \%, 86 \%$, e $33 \%$ ao comando das tarefas -, percebe-se que o Pinte o 7 está no caminho para ser uma ferramenta completa voltada ao ensino de algoritmos e programação; seja pelas facilidades promovidas pela linguagem LOGO utilizada na ferramenta; pelos elementos lúdicos e contextuais no mundo do usuário encontrados na interface; usabilidade, tecnologias livres e de fácil compartilhamento; além do interesse claramente encontrado nos alunos durante o teste aplicado; o P7 se prenuncia a um futuro próximo, como um sistema tangível e viável ao auxílio no ensino-aprendizado de programação para crianças no Brasil.

O aplicativo já está em sua terceira versão, e vem sendo aprofundado atualmente, em duas linhas de pesquisa. Uma de funcionalidades relativas à criação de relatórios de uso da ferramenta, com suporte para cadastro de alunos, professores, tarefas, compartilhamentos e visualização de relatórios de uso. E outra, a propor uma nova interface à ferramenta, com base no design responsivo e suporte para tablets e outros dispositivos móveis.

A busca na melhora qualitativa e quantitativa da ferramenta se faz contínua, no que tange seu objetivo de poder cada vez mais, proporcionar interações que despontem além à relação usuário-ferramenta, possibilitando que a lógica computacional passada por meio do ensino-aprendizagem de algoritmos e programação de computadores, conecte saberes por meio da interação, colaboratividade, e compartilhamento. Espera-se aprofundar esta pesquisa em um projeto de Mestrado.

\section{Referências}

BRASSCOM. Disponível em: http://www.brasscom.org.br/brasscom/Portugues/download.php?cod=353. Acessado em: 10/02/2013.

Nielsen, J.; Loranger, H. Usabilidade na Web: Projetando Websites com Qualidade. Rio de Janeiro: Elsevier, 2007.

Nielsen, J. Usability Engineering. Boston: Academic Press, 1993.

Piva, D. J.; Nakamiti, G. S.; Engelbrecht, A. M.; Bianchi, F. Algoritmos de Programação de Computadores. Rio de Janeiro: Elsevier, 2012.

Romani, Bruno. Alfabetização digital é porta de entrada para o século 21, diz diretor da Mozilla. In: Folha Ilustrada, 01/02/2013. Disponível em: http://www1.folha.uol.com.br/tec/ 1223970-alfabetizacao-digital-e-porta-de-entrada-para-o-seculo-21diz-diretor-da- mozilla.shtml. Acessado em: 13/04/2013.

Xavier, G. F. C. Lógica de Programação. São Paulo: Editora Senac, 1999. 